\title{
AUTONOMÍA, DESCENTRALIZACIÓN Y DESCONCENTRACIÓN EN LA CIUDAD DE BUENOS AIRES
}

\section{Gonzalo Martín Rodríguez}

Licenciado en Sociología, Universidad de Buenos Aires, Argentina (2001). Maestrando en Planificación Urbana y Regional, FADU, UBA (2001- actualidad). Investigador becario, Proyecto PICT 1309484 "Globalización y políticas urbanas. Cambios y desafíos para la gestión local" - Dirección del Proyecto: Arq. Beatriz Cuenya - Centro de Estudios Urbano Regionales (CEUR).

Cuaderno Urbano N 4, pp. 99-116, Resistencia, Argentina, Diciembre 2004 


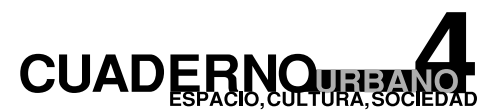

100

Autonomía, Descentralización y Desconcentración en la ciudad de Buenos Aires

\begin{abstract}
Resumen:
El artículo busca examinar algunos aspectos teóricos vinculados al llamado proceso de descentralización territorial operado en la ciudad de Buenos Aires desde 1994 con el logro de su autonomía y, a partir de ella, reconocer cuánto hubo de descentralización y cuánto de desconcentración. Proponemos el análisis del proceso en etapas, haciendo énfasis en la significación implícita que tuvo la eliminación de los Concejos Vecinales, y su reemplazo por los Centros de Gestión y Participación. Sostenemos, como conclusión, que bajo el paraguas de la descentralización se operó, en realidad, una centralización del poder político acompañada de una desconcentración administrativa de funciones subordinadas al poder central. No obstante, el proceso de centralización constituye la condición de posibilidad desde la cual se inicia luego una descentralización social a cuentagotas, en la perspectiva de una verdadera descentralización política que tendrá lugar con la creación de las comunas.
\end{abstract}

\begin{abstract}
:
The article examines some aspects related to the decentralization process operated in Buenos Aires since 1994, when it became an autonomic city. Was it really decentralization or just desconcentration? We study the different periods involved, the implicit meaning of the elimination of the Consejos Vecinales [Neighborhood Councils] that were substituted by the Centros de Gestión y Participación [Management and Participation Centers]. We conclude that instead of decentralization, in fact, a centralization process occurred, accompanied by a desconcentration of administrative extensions of central power. This centralization process has made possible a further, although relative, social decentralization in the perspective if a real politic decentralization which may take place under the establishment of the Comunas [Communes].
\end{abstract}




\section{Gonzalo Martín Rodríguez}

Autonomía, Descentralización y Desconcentración en LA CIUDAD DE Buenos Aires

\section{INTRODUCCIÓN}

En el año 1994 fue reformada la Constitución Nacional Argentina, y se declaró el carácter autónomo de la Ciudad de Buenos Aires. Dos años más tarde, en 1996, sus ciudadanos eligen por primera vez al jefe de gobierno local, al mismo tiempo que se inaugura un proceso de asamblea constituyente que culmina con la sanción de la Constitución de la ciudad. Aquel año fue el puntapié inicial de un proceso al que se ha dado el nombre de descentralización. En Argentina, la descentralización - teniendo en cuenta las distintas variantes de procesos que lícitamente se incluyen bajo este término, tal el caso de Buenos Aires junto al de otras ciudades como Córdoba y Rosario - constituye un novedoso proceso que tiene como objetivo principal la búsqueda de una mejor gobernabilidad en el marco de la globalización, y la crisis económica y política.

El punto de partida de la propuesta descentralizadora es el supuesto de que a lo largo del último medio siglo los estados centrales han acumulado en sus manos cuotas desmesuradas de poder (COPRE; 1989). A partir de allí, surgen las diferentes concepciones y objetivos implícitos y explícitos en la estrategia descentralizadora que actualmente es sostenida por distintos sectores con objetivos distintos y hasta opuestos. Diferentes autores coinciden en señalar que bajo una misma receta suelen estar contenidos al menos dos grandes tipos de propuestas (Coraggio, 1989; De Mattos, 1989; Grillo, 1996; Herzer, 1996). Una de ellas es la perspectiva neoliberal o tecnocrática, para la cual la descentralización constituye un instrumento adecuado para desentender al Estado de la atención de aquellas que históricamente fueron asumidas como sus obligaciones, así como descomprimir la presión popular sobre los niveles centrales del gobierno hacia sus unidades subalternas locales bajo el lema "que cada uno se haga cargo de lo suyo". Otra corriente, en cambio, afirma los aspectos democratizantes y constructores de una ciudadanía basada en la capacidad de control real y de participación de los ciudadanos en la gestión de su hábitat junto con un Estado más próximo, transparente y accesible. El proceso descentralizador de Buenos Aires se sostiene, al menos discursivamente, en esta segunda corriente. 


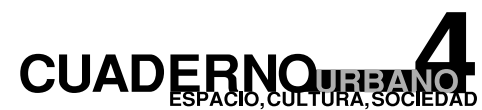

102

Autonomía, Descentralización y Desconcentración en la ciudad de Buenos Aires

1-Alos fines de este trabajo emplearemos indistintamente los términos ceder, delegar, transferir traspasar, etcétera.)
En este trabajo nos proponemos aportar en la dilucidación de algunos aspectos teóricos vinculados al análisis del proceso de descentralización territorial operado en la ciudad de Buenos Aires con el logro de su autonomía y a partir de ella iHa existido realmente una descentralización? ¿O se trató más bien de una desconcentración del aparato estatal? ¿Cuánto hubo de lo uno y de lo otro? En la primera parte del artículo, desarrollamos nuestra propuesta de marco teórico de la descentralización. Esperamos presentar sin ambigüedades la definición de cada uno de los conceptos que trabajaremos, en particular, la diferencia entre descentralizar y desconcentrar. En una segunda parte, describiremos y expondremos a partir de las categorías teóricas nuestra caracterización del modelo de descentralización iniciado en la Ciudad de Buenos Aires desde la década del '90, haciendo también una reseña de sus antecedentes históricos. Finalmente, en el tercer y último apartado, expondremos nuestras reflexiones finales.

\section{DESCENTRALIZACIÓN Y DESCONCENTRACIÓN}

Cuando se habla de descentralización, el primer acuerdo entre quienes hablan de ella (intelectuales, políticos, técnicos) es que no existe una acepción unívoca del término. A nuestro entender, sin embargo, no es tan relevante la existencia de múltiples significados, como sí lo es definir explícitamente cuál de ellos es el que hemos de utilizar. A los fines de este trabajo, definiremos la descentralización en forma genérica como aquel proceso mediante el cual algún nivel central del Estado cede ${ }^{l}$ poder y responsabilidades en manos de otros actores. Existen por lo menos dos grandes tipos de descentralización de naturaleza muy diferente - no excluyente - entre sí. Tal distinción cobra sentido al considerar quiénes son aquellos actores destinatarios de la descentralización.

Un primer tipo de descentralización está dado por aquella que opera un traspaso de poder y responsabilidades en sentido vertical descendente, desde los niveles superiores de la estructura del Estado hacia los niveles inferiores, generalmente unidades subnacionales de naturaleza territorial (provincias, estados, municipios, ciudades, gobiernos locales en ge- 


\section{Gonzalo Martín Rodríguez}

Autonomía, Descentralización y Desconcentración en LA CIUDAD DE Buenos Aires

neral), pero también otros organismos no territoriales, por ejemplo los entes autárquicos; a este tipo denominaremos descentralización estatal. Según la entendemos aquí, la descentralización aplicada al Estado implica generar algún grado variable de autonomía en alguna unidad inferior del árbol decisional del aparato estatal (Coraggio; 1974) Algunos autores prefieren considerar este tipo de descentralización como el único posible² .

En segundo lugar, tenemos aquella descentralización que opera un traspaso de poder y responsabilidades desde el Estado - sin importar el nivel - hacia actores paraestatales, es decir, la sociedad civil (sea los ciudadanos, o sus organizaciones civiles); a este segundo tipo nos referiremos como descentralización social y, más que a la autonomía territorial, se refiere a la medida en que esa autonomía se sostiene en un poder ciudadano basado en la participación. En ciertos casos, particularmente los vinculados al tipo de proceso desarrollado. En el sentido señalado por Roselli (1996), la descentralización social implica "la apertura de nuevos ámbitos institucionales y canales de relacionamiento, estimulando a los vecinos a participar de la gestión comunal".

Al hablar de descentralización es frecuente encontrar referencias a otro concepto, la llamada desconcentración. Algunos autores hablan de desconcentración para referirse a un tipo específico y primitivo de descentralización (Rondinelli; 1981); en otros casos, se entiende una relación de oposición entre ambos conceptos (De Soto; 1987); por último, tenemos aquellas situaciones en las que los dos conceptos son utilizados de forma indistinta, como equivalentes (algo muy frecuente en el discurso político y la redacción legislativa, en particular en la Argentina).

En este trabajo emplearemos el término desconcentración como un proceso estrictamente espacial: el incremento de la presencia de dependencias y/o funciones del Estado distribuidas en el territorio, en detrimento del peso relativo de esa presencia en el área central de la ciudad. Como tal, la desconcentración es un proceso de índole estrictamente espacial,
2- Por ejemplo, Burki, Perry y Dillinger (1999) expresando en gran medida el punto de vista del Banco Mundial. Estos autores plantean además, que para hablar de descentralización debe necesariamente existir un gobierno elegido por el pueblo, lo cual no les impide plantear que la primera etapa de la descentralización ocurrió, en la Argentina, durante el último gobierno de facto. 
Autonomía, Descentralización y Desconcentración en la ciudad de Buenos Aires

3- Agreguemos, además, que la desconcentración es un término hastaciertopunto relativo. Ello nos lo indica claramente el hecho de que el acceso de los ciudadanos a los servicios del Estado puede tender a independizarse cada vez más del espacio. Imaginemos, $a$ modo de ejemplo, una ciudad donde todos los habitantes tienen acceso ilimitado a internet desde susdomicilios, teniendo la posibilidad de realizar por ese medio - y sin efectuar traslado alguno-una serie de trámites que previamente les hubiera requerido trasladarse

físicamente hasta una agencia del Estado. No por ello dejarân de existirunidadesfísicas

localizadas del Estado, pero, ciertamente, ya no sera

fundamental la forma en que las mismas se distribuyanenel territorio de la ciudad. a diferencia de la descentralización que corresponde al ámbito de las relaciones sociales aespaciales.

La desconcentración es aplicable a los fines de este trabajo para caracterizar aquellos procesos de relocalización de funciones del Estado en el territorio, tanto de aquellas funciones que requieren la presencia efectiva del ciudadano en una dependencia estatal a fin de realizar un trámite o participar de una actividad, como aquellas funciones que implican una intervención del Estado en el territorio. En las primeras, el resultante de la desconcentración es que los ciudadanos ya no precisan trasladarse hasta el área central para acceder a los servicios que brinda el Estado, sino que pueden hacerlo realizando un recorrido menor hasta una dependencia más próxima a su lugar de residencia. En el segundo tipo de funciones, la desconcentración implica como resultado una menor distancia relativa de la dependencia localizada respecto del punto más alejado de su área de intervención (por ejemplo una dirección de mantenimiento urbano).

De esta manera, dada una determinada función, la desconcentración puede darse ya sea mediante:

* un incremento del número de dependencias localizadas en puntos del territorio donde la función se desarrolla;

* una relocalización de dependencias, es decir, igual número de localizaciones pero distribuidas de manera más eficiente.

Al hablar de desconcentrar funciones, interesa resaltar que eso no implica, necesariamente, relocalizar el $100 \%$ de su estructura burocrática; una parte más o menos considerable de la burocracia podrá permanecer en el área central (o cualquiera otra área) en la medida que resulte necesario. Por lo demás, agreguemos que la desconcentración no es el único expediente que puede conducir a mejorar el acceso de los ciudadanos al Estado y la fluidez de la relación; existen otras variables políticas, tecnológicas ${ }^{3}$, etcétera. 


\section{Gonzalo Martín Rodríguez}

Autonomía, Descentralización y Desconcentración en LA CIUDAD DE Buenos Aires

\section{Autonomía, descentralización y desconcentración en Buenos Aires}

El denominado proceso de descentralización en la Ciudad de Buenos Aires fue y es, como veremos, un proceso mucho más complejo y rico para el análisis de lo que comúnmente se tiende a creer. Un análisis de este proceso requiere hurgar mucho más profundo y, hasta cierto punto, prescindir de la superficialidad del discurso político que habitualmente es el que impone su sentido al imaginario social que existe acerca de este complejo tema. A continuación, intentaremos describir e interpretar en términos de desconcentración y descentralización los procesos de reforma de la estructura de gobierno de la ciudad de Buenos Aires desde mediados de la década del 90, previa revisión de sus antecedentes más inmediatos.

\section{ANTECEDENTES DE LA DESCENTRALIZACIÓN. LA LEY ORGÁNICA MUNICIPAL}

\section{$\underline{\mathrm{DE}} 1972$}

Antes de alcanzar el estatus de autonomía, la ciudad de Buenos Aires era un territorio federal bajo jurisdicción del Poder Ejecutivo Nacional. Su estructura de gobierno se organizaba en tres niveles. En el primer nivel estaba el intendente, que era un cargo designado directamente por el presidente de la Nación. En un segundo nivel había un Consejo Deliberante de 60 miembros, electos por los vecinos de la ciudad, la cual a tal fin constituía un distrito electoral único. Y había, además, un tercer órgano de gobierno: 14 consejos vecinales que correspondían a cada una de las 14 zonas en que se dividía la ciudad. Cada consejo vecinal se conformaba por 9 vocales que eran electos por los vecinos de las respectivas zonas. Esta estructura de gobierno, las respectivas atribuciones de cada nivel y demás formalidades se hallaban establecidas mediante la ley 19.887, más conocida como Ley Orgánica de la Municipalidad de la Ciudad de Buenos Aires, sancionada en el año 1972.

Viene al caso una observación. Cuando hablamos de que la ciudad de Buenos Aires no era autónoma, estamos hablando de una ciudad que, sin embargo, no era la ciudad mártir. Los vecinos de Buenos Aires, además de elegir sus concejales y vocales que los representaban a 
Autonomía, Descentralización y Desconcentración en la ciudad de Buenos Aires

nivel de la ciudad y el barrio, también elegían, como cualquier provincia, sus representantes a las Cámaras de diputados y senadores nacionales. Desde luego, también computaban sus votos para la elección del presidente de la República. Por otro lado, la Capital Federal no era precisamente uno de los territorios olvidados de la patria; dentro de sus límites funcionaba probablemente la mejor oferta de bienes, servicios, infraestructuras y, por supuesto, los niveles de empleo, de alfabetismo, de mortalidad infantil, el ingreso per cápita, y cuanto indicador de calidad de vida se nos pudiera ocurrir (salvo quizás los de indicadores de delito y contaminación) eran los mejores del país. Era, además, la vidriera de la Argentina: todo lo que en Buenos Aires sucedía, tenía una repercusión amplia e inmediata en el resto del territorio y la opinión pública nacional. En definitiva, aunque en lo formal la ciudad no era autónoma, sí era, sin dudarlo, una ciudad con poder.

Es interesante recordar que, aunque hoy parezca algo olvidado, en aquella Ley Orgánica de 1972 ya se hacía referencia al concepto de descentralización, aunque el empleo del término ya entonces se confundía con el de desconcentración. Así, vemos que era una atribución del Consejo Deliberante "promover la descentralización de los servicios transfiriendo funciones y asignando los recursos pertinentes a los Consejos Vecinales" (Art. 9) y que era una función del intendente municipal "Atender la prestación de los servicios públicos propendiendo a una adecuada descentralización” (Art. 31)

Según la ley, los consejos vecinales tenían entre sus atribuciones: colaborar con el nivel central en la formulación de proyectos de interés comunal y opinar sobre los proyectos que el nivel central ponga en su conocimiento; ejecutar obras con financiación directa del vecindario; prestar servicios desconcentrados; mantenimiento de plazas y paseos, cercos y veredas, la recolección de residuos, barrido y limpieza, la inspección de higiene, y la policía de moral y buenas costumbres; controlar y formular observaciones relativas a proyectos y obras del gobierno central; elevar al intendente su presupuesto anual de gastos y recursos, y otras atribuciones. 


\section{Gonzalo Martín Rodríguez}

Autonomía, Descentralización y Desconcentración en LA CIUDAD DE Buenos Aires

También, los consejos vecinales debían promover la participación de la población en el progreso material, moral y cultural del vecindario, podían considerar proyectos presentados por las organizaciones de la comunidad, y hasta estaban facultados para desarrollar proyectos y obras locales con financiamiento voluntario de los vecinos o de consorcios autorizados.

Hacia el año $1985^{4}$, funcionaba de forma desconcentrada, en los Consejos Vecinales, el Registro Civil. En ese año, se desconcentra también la Dirección General de Rentas cuyas oficinas, antes de ser relocalizadas, funcionaban en sucursales bancarias. Al poco tiempo, se crea también el Servicio Social Zonal, encargado de la implementación de planes de asistencia social. A decir verdad, este último no fue desconcentrado, puesto que no existía antes.

Generalmente, se admite que la estructura territorial del gobierno de Buenos Aires era, en el nivel de los Consejos Vecinales, formalmente una estructura descentralizada (Pírez; 1996, Grillo; 1996). Sin embargo, no se han producido hasta hoy esfuerzos significativos por comprender la naturaleza de aquella experiencia, los motivos de su supuesto fracaso y, menos aún, las causas de su desaparición. En el debate sobre la descentralización, las referencias a los consejos vecinales son siempre superficiales, sin profundizar en el análisis de las causas del fracaso y su posterior eliminación una vez - paradójicamente - alcanzada la autonomía de la ciudad. Chiernajowsky (1998) sugiere, como hipótesis explicativa del fracaso, que los consejos vecinales eran "instituciones vacías y carentes de autonomía" por cuanto "carecían de poder de control, poder de gestión, y verdadero poder político".

Aún admitiendo la hipótesis del fracaso en términos de la ausencia de "poder real", quedan planteados por lo menos dos interrogantes. Primero, si allí no había poder ipor qué los partidos políticos se disputaban la representación en los consejos vecinales? Y segundo ipor qué no se los conservó y se les dio el poder que necesitaban? En lugar de ello, se los eliminó. Como veremos más adelante, se impusieron en su lugar nuevas instituciones (los CGP) que no se caracterizarían, justamente, por su autonomía. $\overline{4-\text { Durante la dictadura militar de }}$ 1976-1983 los consejos vecinales (así como el Consejo Deliberante) fueron cerrados, y la mayoría de sus funciones desconcentradas fueron "reconcentradas" en el área central de la ciudad. 


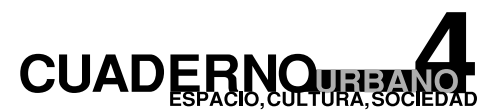

108

Autonomía, Descentralización y Desconcentración en la ciudad de Buenos Aires

5-Nofueronestos losúnicos puntos que formaron parte de aquel Pacto, pero sílos más relevantes en lo que hace al tema de este trabajo.

\section{4-1996. Pacto de Olivos, Reforma Constitucional y Autonomía}

El proceso que desemboca en la autonomía de Buenos Aires tiene su origen en 1994, cuando las dos principales fuerzas políticas nacionales - PJ y UCR - acuerdan el denominado Pacto de Olivos para reformar la Constitución Nacional. Los términos del acuerdo eran fundamentalmente dos: el Partido Justicialista reclamaba la posibilidad de reelección del entonces presidente Carlos Menem (con amplias posibilidades de ganar, como de hecho sucedió) y el partido radical exigía la autonomía de la Ciudad de Buenos Aires (cuyo electorado, históricamente, le había sido favorable). El resultado que se esperaba era: Menem sería reelecto presidente por el justicialismo, y un radical accedería al gobierno de la más importante ciudad del país ${ }^{5}$, dando lugar a una novedosa situación de convivencia política entre dos fuerzas históricamente rivales. Conviene resaltar que más allá de cualquier interpretación sobre el carácter espurio de aquel acuerdo, los fundamentos políticos, teóricos e ideológicos eran bastante fuertes, y tanto los sectores intelectuales como la opinión pública en general - y más allá de los oportunos cuestionamientos- expresaban un consenso. De cualquier manera, la reforma de la Constitución que posibilitaba el logro de la autonomía - más allá de un acto de justicia - no fue tanto la concreción de un viejo y sentido anhelo de sus habitantes (por el cual, al fin y al cabo, nunca se reclamó activamente) como el fruto de un acuerdo superestructural llevado adelante por la clase política.

Firmado el acuerdo, en junio de 1996, dos años después de la Reforma de la Constitución Nacional, los vecinos de la ciudad eligen por primera vez su jefe de gobierno. También eligieron a los representantes (llamados estatuyentes) que tuvieron a su cargo en los meses siguientes la redacción de la Constitución de la Ciudad Autónoma de Buenos Aires, la que finalmente se aprueba en octubre de ese mismo año. El artículo 140, intitulado Cláusula Derogatoria, procede a derogar todas las normas preexistentes que pudieran oponerse a lo establecido por la Constitución. De esta manera, caducan todas las disposiciones de la ley 19.887 de 1972, y desde aquel preciso momento los consejos vecinales dejan de existir. 


\section{Gonzalo Martín Rodríguez}

Autonomía, Descentralización y Desconcentración en LA CIUDAD DE Buenos Aires

¿Qué cambios fundamentales implica en la estructura de gobierno de la ciudad de Buenos Aires la nueva Constitución? En primer lugar, interesa destacar que se conserva el mismo número de niveles, modificándose, por cierto, sus denominación y atribuciones. En el primer nivel existe desde entonces un jefe de gobierno electo (que reemplaza la figura del intendente designado); en el segundo nivel, una Legislatura (que reemplaza al anterior Consejo Deliberante); y por último, hay un tercer nivel constituido por una cantidad no especificada de comunas "a futuro" (sustitutas de los anteriores consejos vecinales)

1996-1998. Primera etapa de la autonomía: desconcentración y consolidación del poder central

El primer gran desafío del nuevo gobierno autónomo era constituirse en un verdadero centro de gestión. Como señala Pírez $(1996,183)$ hablar de descentralización supone "diferenciar un centro decisional con competencias sobre el conjunto del territorio del gobierno local (...) En la ciudad de Buenos Aires, podemos afirmar, no existe ese centro de gestión”. Un proceso de descentralización en el territorio es, a la vez, un proceso de generación de una o varias centralidades nuevas, que reciben de pronto una cantidad de competencias para las cuales, sin embargo, deben desarrollar todo un proceso de aprendizaje y de reorganización institucional.

Pero en el caso de la naciente ciudad autónoma, no bastaba con transferir ese poder desde arriba. En efecto, el nuevo gobierno local autónomo se constituye en primer término, a partir de una cuota de poder que le es transferido desde el Estado Nacional; pero en segundo término necesitaba asegurar un control político del territorio tal que le permitiera ejercer ese poder que estaba recibiendo sin mayores inconvenientes. No nos referimos estrictamente al control policial del territorio, una competencia que, por lo demás, estuvo ausente de la Reforma constitucional, y su ejercicio aún hoy sigue en manos de la policía federal. Más bien, nos referimos al ejercicio del poder político, ya sea a través de redes clientelares como 
Autonomía, Descentralización y Desconcentración en la ciudad de Buenos Aires

de espacios de disputa política y representación concreta de los vecinos como eran los consejos vecinales.

Es en este sentido que, a nuestro juicio, la desaparición de los consejos vecinales no se explica tanto a su supuesto fracaso, como por la necesidad de generar las condiciones que hicieran posible la constitución de un gobierno local autónomo en Buenos Aires en el corto plazo y con el menor grado de disputa posible. Los consejos vecinales son descartados porque la descentralización se presentaba como el punto de llegada y no como el punto de partida en aquel difícil proceso, en un contexto político también complejo. Mantenerlos conservando su dosis de autonomía (y peor aún, incrementándola) probablemente hubiera significado para el nuevo gobierno tener que "compartir el poder" sobre el territorio con otras fuerzas políticas, lo que no resultaba muy aconsejable, ni deseable, puesto que a nadie en general le agrada compartir el poder.

De haberse conservado y reformado los consejos vecinales, sin modificar los grados de autonomía con la que ya contaban (elección de los vocales por los vecinos) iqué implicancias hubiera tenido para el nuevo gobierno autónomo? La respuesta nos la da el propio jefe del primer gobierno autónomo de la ciudad. Unos meses antes de ganar la Jefatura de Gobierno, el entonces senador Fernando de la Rúa exponía a la intelectualidad presente, en el Seminario Internacional Descentralización y Gobiernos Locales, su proyecto para la organización del gobierno de la ciudad. Nos permitimos aquí realizar una larga cita de los aspectos centrales de aquel proyecto. De la Rúa (1996; 181-182) hablaba de la necesidad de crear

"una instancia de gobierno más próxima al pueblo, a la gente (...) estamos hablando de los principios de la desconcentración administrativa, a través de los denominados Centros de Gestión Vecinal (...) debe descartarse la posibilidad de establecer mecanismos de descentralización política (...) La dispersión del poder político, de la unidad del mando administrativo y la transferencia integral de las funciones del Estado a zonas descentralizadas, tratán- 


\section{Gonzalo Martín Rodríguez}

Autonomía, Descentralización y Desconcentración en LA CIUDAD DE Buenos Aires

dose de una zona geográfica pequeña y sumamente interrelacionada, generaría un verdadero caos en el gobierno autónomo (...) El camino que proponemos es el de organizar el régimen municipal sobre la base de mantener la centralización normativa y el ejercicio del poder político en la Legislatura de la Ciudad y su jefe de gobierno; pero al mismo tiempo, estableceremos la desconcentración y transferencia de funciones ejecutivas hacia los organismos que denominaremos Centros de Gestión Vecinal" (bastardillas nuestras).

Aquellas palabras del futuro jefe de gobierno, poniendo blanco sobre negro en las diferencias entre descentralizar (compartir poder) y desconcentrar (extender poder propio en el territorio), revelaban por cierto una asombrosa claridad teórica y política. No obstante, aquel proyecto original de De la Rúa, que hablaba de "desconcentrar, no descentralizar", contrastaría notablemente - al menos en apariencia - con el espíritu que meses más tarde quedaría expresado en la redacción final de la nueva Constitución, y que tomaría forma definitiva en el Título Sexto referido a las futuras comunas. Mientras De la Rúa prometía "desconcentrar, no descentralizar", la Constitución hablaba claramente de "descentralizar", y el término "desconcentrar" estaba literalmente ausente del texto.

Aunque parecían correr por caminos diferentes, esta contradicción entre ambos proyectos era tan sólo aparente. Apenas asumida la Jefatura de Gobierno, De la Rúa decreta la elaboración de un Programa de Descentralización Administrativa. Unos meses más tarde, crea la Subsecretaría de Descentralización. ¿Qué ocurrió en el medio? Simplemente, un cambio de discurso. Como veremos a continuación, el proyecto original de "desconcentrar, no descentralizar" se mantuvo en gran medida intacto. La misma Constitución de la ciudad establecía que las comunas descentralizadas debían crearse por ley en un plazo no menor de cuatro años y no mayor de cinco". Durante ese lapso, el nuevo gobierno autónomo podía disponer para sí de la vieja estructura de los consejos vecinales, con el compromiso de, luego de ese período, abrir nuevamente el juego a la disputa de otras fuerzas con la creación de las comunas. 


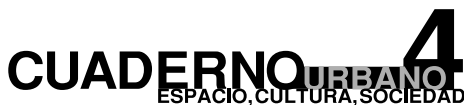

Autonomía, Descentralización y Desconcentración en la ciudad de Buenos Aires

6- Los CGP son los CGV del proyecto original de De la Rúa. El concepto de la "participación" también era un principio

claramente establecidoenel mandato constitucional

7-Elproceso institucional cuenta con ciertos vicios como ser que la creación de los CGP (en septiembre) es anterior a la sanción de la nueva Constitución (octubre) y, por ello, previa a la desaparición formal de los consejosvecinales.
Es así como en septiembre de 1996 mediante el decreto 213, se crean los Centros de Gestión y Participación ${ }^{6}$, que comienzan a funcionar en las mismas instalaciones donde anteriormente se ubicaban los consejos vecinales. Las jurisdicciones territoriales correspondientes a cada CGP también son las mismas, con la salvedad de que en lugar de catorce zonas pasan a constituirse dieciséis, al subdividirse dos de aquellas que abarcaban las áreas más densas de la ciudad. Resumiendo, 16 CGP en lugar de 14 Consejos Vecinales ${ }^{7}$. Comparados con sus predecesores, si los consejos vecinales contaban con cierta autonomía formal, los CGP no cuentan con grado de autonomía alguno de autonomía, ni siquiera formal. En este sentido, expresan claramente el proyecto original del jefe de gobierno radical. Respecto de su estructura organizativa, los CGP cuentan con un cargo de director, que es designado directamente por el Poder Ejecutivo sin requerir acuerdo ni consideración por parte de la Legislatura. En un principio, comienzan funcionando en los CGP prácticamente los mismos servicios desconcentrados que se brindaban antes en los consejos vecinales.

\section{8-2003. Segunda etapa de la autonomía: descentralización a cuentagotas}

Hasta aquí nos hemos referido exclusivamente, por un lado, al tipo de descentralización que denominamos estatal y a la desconcentración territorial del aparato del Estado. En la segunda etapa de la autonomía de la ciudad, se introduce, a nuestro juicio, un nuevo proceso: la descentralización social. Habíamos definido la descentralización social como aquel proceso que verifica un traspaso de poder desde el Estado — como totalidad, más allá de sus niveles- hacia su afuera, la sociedad civil.

A partir de 1998, los Centros de Gestión y Participación incorporan una batería de funciones desconcentradas que se agregan a las existentes: Sistema informático de reclamos vecinales (SUME), renovación de licencias de conducir, servicio de mediación comunitaria, defensoría del consumidor, servicios de asesoría jurídica gratuita, justicia de faltas, abono de infracciones de tránsito, otorgamiento de permisos de venta en la vía pública y ferias artesanales, programación de eventos culturales, control de residuos domiciliarios, mantenimiento urbano y obras conexas, recepción de denuncias de infracciones al código de 


\section{Gonzalo Martín Rodríguez}

Autonomía, Descentralización y Desconcentración en LA CIUDAD DE Buenos Aires

planeamiento y de edificación, otorgamiento de permisos de obras particulares, y un sistema de ventanilla única, que aporta a mejorar la eficiencia en la atención al público. No obstante, desde ese año, se refuerza la injerencia del nivel central en los CGP al incorporar un nuevo funcionario: el delegado comunal, quien aunque su denominación pareciera connotar otra cosa, no es un delegado de la comunidad sino del Poder Ejecutivo ante la comunidad.

Por otro lado, haciendo cierto honor a su nombre, los CGP comienzan a incorporar un nuevo proceso: la participación comunitaria. Por medio del decreto 1958, del todavía jefe de gobierno De la Rúa, se crea en el ámbito de los CGP el Registro de Organizaciones de Acción Comunitaria (ROAC). Las organizaciones allí inscriptas se encuentran autorizadas para participar de los llamados Consejos Consultivos Honorarios $(\mathrm{CCH})$ "cuya función será la presentación de iniciativas y propuestas, información y seguimiento de las prestaciones públicas". En la práctica, sin embargo, pocas organizaciones de la comunidad logran cumplir con los exigentes requisitos que se les impone.

Dos años después, luego de un período en el que se van consolidando la organización y prestación de los servicios desconcentrados y los mecanismos de participación, se produce en 2002 un verdadero salto cualitativo en la descentralización social. En aquel año, por primera vez se hace efectivo el principio del presupuesto participativo establecido en el artículo 52 de la Constitución. Aunque de carácter realmente limitado, puesto que sólo se somete a discusión el 3\% del presupuesto de la ciudad, los vecinos de la ciudad y organizaciones por primera vez pueden involucrar su opinión y su voto en la definición de las áreas y proyectos de asignación presupuestaria prioritarios, en función de las necesidades de su barrio. Sin embargo, al cabo de tres años consecutivos, se puede afirmar que el éxito ha sido bastante relativo: la participación de los vecinos es escasa, la última palabra sobre la distribución del presupuesto la continúan teniendo las oficinas centrales, y, por lo demás, las asambleas del presupuesto participativo se prestan a manejos de tipo clientelares.

En el mismo sentido del presupuesto participativo y en el marco de lo que denominamos descentralización social, se efectiviza también en 2002 el llamado Plan de Prevención del 


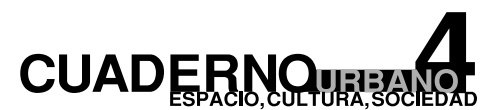

114

Autonomía, Descentralización y Desconcentración en la ciudad de Buenos Aires

Delito (PPD). El objetivo principal de este programa es establecer la cooperación del Estado y la comunidad del barrio en la construcción del mapa del delito y el diseño de programas y estrategias de prevención.

En síntesis, el período 1998 hasta la actualidad ha estado signado por una consolidación y profundización del proceso desconcentrador. A cuentagotas, y con evidentes limitaciones no necesariamente vinculadas con una falta de voluntad política de los impulsores, también se ha comenzado a desarrollar un proceso de descentralización social. A la espera de ser tratados en la Legislatura de la ciudad, existe casi una treintena de proyectos de Ley Comunas, a pesar de que hace dos años ha vencido el plazo establecido por la Constitución.

\section{$\underline{\text { CONCLUSIONES }}$}

A partir de la Reforma de la Constitución de 1994, la sanción de la Constitución de la Ciudad Autónoma de Buenos Aires en 1996, y la elección del primer jefe de gobierno, tenemos un país que se ha descentralizado un poco más. Sin embargo, en términos estrictamente teóricos, no tenemos, al menos por ahora, una ciudad descentralizada. La estructura descentralizada que, con sus evidentes limitaciones, existía antes de la eliminación de los consejos vecinales, ha cedido su lugar a una estructura de tipo centralizada y desconcentrada. Tal era el contenido del proyecto original de radicalismo, al que hemos resumido como una propuesta de "desconcentrar, no descentralizar".

La hipótesis que podría explicar este hecho es la dificultad de desarrollar un proceso de descentralización en ausencia de un nivel previo de centralidad. El proceso de centralización/desconcentración, abierto en 1996, habría tenido la funcionalidad de consolidar el poder de control sobre el territorio que precisaba el nuevo gobierno autónomo. De esta manera, se explicaría la desaparición de los anteriores consejos vecinales que, de alguna manera, hubieran conspirado contra la gobernabilidad de la ciudad según el proyecto radical. 


\section{Gonzalo Martín Rodríguez}

Autonomía, Descentralización y Desconcentración en LA CIUDAD DE Buenos Aires

El - tantas veces - proclamado proceso de descentralización no fue tal. Un gobierno central que, si se permite la metáfora, ha multiplicado sus ojos y manos en el territorio podrá ser, ciertamente, un Estado más eficiente, transparente, visible, etcétera. Pero no es, estrictamente hablando, un Estado descentralizado. Descentralizar, recordémoslo, implica necesariamente que en algún punto y en alguna medida, se rompa una cadena de mando. Si esta ruptura es total, estamos hablando de independencia, y si es parcial, hablamos de autonomía, semi-autonomía, autarquía, etcétera. En la práctica, podrá resultar las más de las veces complicado y difusa la frontera entre desconcentrar y descentralizar, más aún cuando ambos procesos se desarrollan de forma paralela y hasta complementaria, apoyándose mutuamente. Inclusive, no sería descabellado afirmar que todo acto de desconcentración implica, aún sin pretenderlo, cierto grado de descentralización aunque sea mínimo. No obstante, en el caso de Buenos Aires, afirmamos que mediante el bautizado "proceso de descentralización" se ha buscado consolidar y extender territorialmente el poder central, antes que redistribuirlo.

La redistribución efectiva del poder, en la variante de la descentralización social, comienza a verificarse a cuentagotas recién en los últimos años, al implementarse mecanismos de participación comunitaria en la gestión pública y social del territorio como los Consejos Consultivos, el Plan de Prevención del Delito y el Programa de Presupuesto Participativo. La descentralización estatal, en cambio, que supondría mayor autonomía de las subunidades territoriales del Estado, aún no se ha producido, a la espera de la sanción de la Ley de Comunas cuyo plazo ya se ha vencido hace más de dos años.

Con toda su complejidad, que sólo en parte hemos logrado abordar en este trabajo, el logro de la autonomía y la experiencia descentralizadora de Buenos Aires constituyen dos elementos de un proceso que no ha alcanzado, al menos todavía, el lugar que debiera corresponderle en aquellas disciplinas que tienen por objeto el estudio de los procesos sociales en el territorio. En ese sentido, esperamos haber realizado nuestro aporte. En particular, creemos además, se abre una interesante línea de estudio acerca de la eficacia de la descentralización en cuanto al logro y verificación empírica de sus postulados políticos y teóricos. 


\section{CUADERNA}

Autonomía, Descentralización y Desconcentración en la ciudad de Buenos Aires

\section{$\underline{\text { BIBLIOGRAFÍA }}$}

Burki, Perry y Dillinger (1999) Más allá del Centro: la descentralización del Estado. Banco Mundial. Chiernajowsky, L. (1998) Discurso pronunciado en la apertura del seminario "La Descentralización y Modernización del Gobierno Local", Edición preliminar, Vicejefatura de Gobierno del GCBA.

GCBA (1999) La Descentralización. Publicación del Programa de Descentralización.

Coraggio, J. L. (1989) La Propuesta de la descentralización: en busca de un sentido popular; en Descentralización del Estado, requerimientos y políticas en la crisis. Laurelli, E. y Rofman, A. (comp.) Ediciones CEUR. Coraggio, J. L. (1974) Concentración espacial y centralización; discusión de algunas falacias. CEUR-ITDT, Buenos Aires.

De Mattos, C. (1989) La Descentralización zuna nueva panacea para enfrentar el subdesarrollo regional? en "Descentralización del Estado, requerimientos y políticas en la crisis” Laurelli, E. y Rofman, A. (comp.) Ediciones CEUR De Soto, H. (1987) El Otro Sendero. Editorial Sudamericana, Buenos Aires.

COPRE, Comisión para la Reforma del Estado de Venezuela (1989) La Descentralización. Caracas.

Pírez, P. (1996) Descentralización y gestión de la ciudad de Buenos Aires; en "Ciudad de Buenos Aires: Gobierno y Descentralización". Herzer H. (comp.), Ediciones CEA-CBC.

Grillo, O. (1996) Descentralización: contraindicaciones para su uso en la ciudad de Buenos Aires; en "Ciudad de Buenos Aires: Gobierno y Descentralización”. Herzer H. (comp.), Ediciones CEA-CBC.

De la Rúa, F. (1996) La organización del gobierno autónomo de la Ciudad de Buenos Aires; en "Ciudad de Buenos Aires: Gobierno y Descentralización". Herzer H. (comp.), Ediciones CEA-CBC.

Roselli, A. (1996) Experiencia de descentralización en la ciudad de Montevideo, Uruguay; en "Ciudad de Buenos Aires: Gobierno y Descentralización". Herzer H. (comp.), Ediciones CEA-CBC.

Rondinelli, D. (1981) Government Decentralization in Comparative Perspective: Theory and Practice in developing Countries, en "International Review of Administrative Science" № 47.

Constitución de la Ciudad Autónoma de Buenos Aires (1996).

Constitución de la República Argentina (1994). 